\title{
Lagrangian geometry of the Gauss images of isoparametric hypersurfaces in spheres
}

https://doi.org/10.1515/coma-2019-0013

Received February 8, 2019; accepted May 20, 2019

Abstract: The Gauss images of isoparametric hypersufaces of the standard sphere $S^{n+1}$ provide a rich class of compact minimal Lagrangian submanifolds embedded in the complex hyperquadric $Q_{n}(\mathbb{C})$. This is a survey article based on our joint work [17] to study the Hamiltonian non-displaceability and related properties of such Lagrangian submanifolds.

Keywords: isoparametric hypersurfaces, monotone Lagrangian submanifolds, Floer homology

MSC: Primary: 53C40; Secondary: 53C42, 53D12

\section{Introduction}

This is a survey article based on our joint work [17]. The aim of our work is to build a bridge between the symplectic geometry and the submanifold theory. Here by symplectic geometry, we mean the Floer theory for Lagrangian intersections, and by submanifold theory, isoparametric hypersurface theory. The isoparametric hypersurface has been well-investigated in submanifold theory and it has several nice structures and properties not only in differential geometry but also from the viewpoint of differential topology, Lie theory, partial differential equations, integrable systems and mathematical physics.

The Floer theory has been well-developed, but not much is known in concrete cases. The Gauss images (the images of the Gauss map) of isoparametric hypersurfaces in the standard sphere $S^{n+1}$ supply a rich class of compact minimal Lagrangian submanifolds embedded in the complex hyperquadric $Q_{n}(\mathbb{C})$. When $n=1$ such a Gauss image is nothing but a great circle of the standard 2-sphere. We study the Lagrangian intersection theory on this class.

For a Lagrangian submanifold $L$ of a symplectic manifold $(M, \omega)$, consider a Hamiltonian diffeomorphism $\varphi$ of $M$ with transverse intersection $L \cap \varphi(L)$. To give a lower bound of the number $\sharp(L \cap \varphi(L))$ of intersection points is an important and difficult problem, which is the so-called Arnold inequality. Floer has invented the Floer (co)homology to solve this problem. In this article a symplectic manifold, which we are concerned with, is the complex hyperquadrics

$$
Q_{n}(\mathbb{C}):=\left\{[\mathbf{z}] \in \mathbb{C} P^{n+1} \mid z_{0}^{2}+z_{1}^{2}+\cdots+z_{n+1}^{2}=0\right\} \subset \mathbb{C} P^{n+1}
$$

or the real Grassmann manifolds of oriented 2-planes

$$
\begin{aligned}
& \widetilde{G r}_{2}\left(\mathbb{R}^{n+2}\right)\left(\subset \wedge^{2} \mathbb{R}^{n+2}\right) \\
& :=\left\{[W] \mid \text { oriented 2-dimensional vector subspaces of } \mathbb{R}^{n+2}\right\}
\end{aligned}
$$

\footnotetext{
`Corresponding Author: Reiko Miyaoka: Mathematical Institute, Tohoku University, Sendai 980-8578, Japan, E-mail: r-miyaok@tohoku.ac.jp

Yoshihiro Ohnita: Osaka City University Advanced Mathematical Institute, \& Department of Mathematics, Osaka City University, 3-3-138 Sugimoto, Sumiyoshi-ku, Osaka 558-8585, Japan, E-mail: ohnita@sci.osaka-cu.ac.jp
} 
which are identified through the diffeomorphism

$$
\widetilde{\mathrm{Gr}}_{2}\left(\mathbb{R}^{n+2}\right) \ni[W]=\mathbf{a} \wedge \mathbf{b} \longleftrightarrow[\mathbf{a}+\sqrt{-1} \mathbf{b}] \in Q_{n}(\mathbb{C})
$$

where $\{\mathbf{a}, \mathbf{b}\}$ is an orthonormal basis of $[W]$ compatible with its orientation. This manifold has the homogeneous space expression

$$
Q_{n}(\mathbb{C}) \cong \widetilde{G r}_{2}\left(\mathbb{R}^{n+2}\right) \cong S O(n+2) / S O(2) \times S O(n)
$$

which is a compact Hermitian symmetric space of rank 2. Our Lagrangian submanifolds in $Q_{n}(\mathbb{C})$ is the image of the Gauss map of an oriented hypersurface immersed in the unit standard sphere $S^{n+1}=S^{n+1}(1)$

$$
N^{n} \hookrightarrow S^{n+1} \subset \mathbb{R}^{n+2},
$$

where the Gauss map is defined by

$$
\mathcal{G}: N^{n} \ni p \longmapsto \boldsymbol{x}(p) \wedge \boldsymbol{n}(p)=[\boldsymbol{x}(p)+\sqrt{-1} \boldsymbol{n}(p)] \in Q_{n}(\mathbb{C}),
$$

for the position vector $\boldsymbol{x}$ of points on $N^{n}$ and the unit normal vector field $\boldsymbol{n}$ of $N^{n}$ in $S^{n+1}(1)$. We remark the following properties (cf. [34], [19]):

Proposition 1.1. The Gauss map $\mathcal{G}$ of $N$ is a Lagrangian immersion.

Proposition 1.2 ([19]). A deformation of $N^{n}$ in $S^{n+1}$ gives a Hamiltonian deformation of 9 . Conversely a small Hamiltonian deformation of $\mathcal{G}$ is given by a deformation of $N^{n}$ in $S^{n+1}(1)$.

In this article we consider isoparametric hypersurfaces of $S^{n+1}$ as oriented hypersurfaces $N$.

This article is organized as follows: In Section 2 we recall the fundamental theory of isoparametric hypersurfaces in the standard sphere and the properties of the Gauss images of such isoparametric hypersurfaces as Lagrangian submanifolds in complex hyperquadrics. In Section 3 we review some fundamental materials from symplectic geometry and Morse homology. In Section 4 we briefly explain Floer's theory of Lagrangian intersection and in Section 5 we mention the generalization of Floer homology by Y.G. Oh to monotone Lagrangian submanifolds. In Section 6 we explain our results of [17] which discuss the Floer homology of the Gauss images of isoparametric hypersurfaces and study their Hamiltonian non-displaceability. In Section 7 we give the strategy of our proof and remark some related results. In Section 8 we mention a relation of our work to the FOOO theory and Z.Z. Tang's result determining the existence of spin structures on the Gauss images of isoparametric hypersurfaces. In Section 9 we provide some open problems and conjecture.

\section{Isoparametric hypersurfaces in spheres}

Isoparametric hypersurfaces have its origin in the geometric optics in the early 20th century in Italy (Somigliana, Segre, Levi-Civita). They are wave fronts developing according to the Huygens principle.

Let $M$ be a Riemannian manifold with the Levi-Civita connection $\nabla$ and the Laplace operator $\Delta$.

Definition. A $C^{2}$ function $f: M \rightarrow \mathbb{R}$ is called an isoparametric function when $f$ satisfies

(1) $|\nabla f|^{2}=a(f)$

(2) $\Delta f=b(f)$

for some functions $a, b$ on $\mathbb{R}$. A regular level set of an isoparametric function is called an isoparametric hypersurface.

Here, (1) means that the level sets are mutually parallel, and under this condition, (2) means that each level set has constant mean curvature.

Theorem 2.1 ([3]). (1) An isoparametric hypersurface $N$ in the space forms $\mathbb{R}^{n+1}, S^{n+1}$ and $\mathbb{H}^{n+1}$ is a hypersurface with constant principal curvatures, and the converse also holds. 
(2) An isoparametric hypersurface in $\mathbb{R}^{n+1}$ or $\mathbb{H}^{n+1}$ is either totally umbilic or a tube over a totally geodesic submanifold. The number $g$ of distinct principal curvatures is either 1 or 2 .

(3) In $S^{n+1}$, there happen more examples.

In fact, let $\kappa_{1}>\cdots>\kappa_{g}$ be the principal curvatures of $N$ in $S^{n+1}$ and denote by $m_{1}, \ldots, m_{g}$ their multiplicities, respectively.

Theorem 2.2 ([25]). (1) $g \in\{1,2,3,4,6\}$ and $m_{i}=m_{i+2}(i \bmod g)$.

(2) $N$ is given as a level set in $S^{n+1}$ of the so-called Cartan-Münzner polynomial $F$ on $\mathbb{R}^{n+2}$ of degree $g$, which is a real homogeneous polynomial of degree $g$ satisfying two PDE's:

$$
\begin{cases}|\nabla F|^{2} & =g^{2} r^{2 g-2} \\ \Delta F & =c r^{g-2}\end{cases}
$$

where $c=\frac{\left(m_{2}-m_{1}\right) g^{2}}{2}, r=|x|$ for $x \in \mathbb{R}^{n+2}$, and $\nabla$ and $\Delta$ are Euclidean operators.

Thus, any isoparametric hypersurface in the standard sphere is algebraic. Among the level sets, there always exist two lower dimensional level sets, called the focal submanifolds and denoted by $N_{ \pm}$.

A submanifold of $S^{n+1}$ or $Q_{n}(\mathbb{C})$ is called homogeneous if it is given as an orbit under a Lie subgroup of the isometry group $S O(n+2)$.

\section{Example 2.3 :}

- Principal orbits of the isotropy representations of Riemannian symmetric pairs $(U, K)$ of rank 2 . These exhaust all homogeneous isoparametric hypersurfaces in spheres ([15], [35]).

- Algebraic construction of Cartan-Münzner polynomials by representations of Clifford algebras in the case $g=4$ ([33], [11]). These are called of OT-FKM type, among which there exist infinitely many nonhomogeneous isoparametric hypersurfaces.

\subsection{Classification of isoparametric hypersurfaces}

The Classification of isoparametric hypersurfaces in spheres has been completed as follows (2019) :

$g=1: N^{n}=S^{n}$, hyperspheres

$g=2: N^{n}=S^{m_{1}}\left(r_{1}\right) \times S^{m_{2}}\left(r_{2}\right)$, Clifford hypersurfaces

$\left(n=m_{1}+m_{2}, 1 \leq m_{1} \leq m_{2} \leq n-1, r_{1}^{2}+r_{2}^{2}=1\right)$

$g=3: N^{n}=\frac{S O(3)}{\mathbb{Z}_{2}+\mathbb{Z}_{2}}, \frac{S U(3)}{T^{2}}, \frac{S p(3)}{S p(1)^{3}}, \frac{F_{4}}{\operatorname{Spin}(8)}$, Cartan hypersurfaces ([3]).

$g=4: N^{n}$ is either homogeneous or OT-FKM type ([4], [5],[6],[7]).

$g=6:\left(m_{1}, m_{2}\right)=(1,1): N^{n}=\frac{S O(4)}{\mathbb{Z}_{2}+\mathbb{Z}_{2}}([10])$.

$$
\left(m_{1}, m_{2}\right)=(2,2): N^{n}=\frac{G_{2}}{T^{2}}([23],[24])
$$

\subsection{Cohomology of isoparametric hypersurface $N^{n}$}

Let $N_{ \pm}$be the focal submanifolds of $N^{n}$, where $\operatorname{dim} N^{+}=m_{1}+2 m_{2}$ and $\operatorname{dim} N^{-}=2 m_{1}+m_{2}$. Here let the ring of coefficients be

$$
R= \begin{cases}\mathbb{Z} & \text { if } N_{+} \text {and } N_{-} \text {are both orientable } \\ \mathbb{Z}_{2} & \text { otherwise. }\end{cases}
$$

Let $\mu=m_{1}+m_{2}$. 
Theorem 2.4 ([25]).

$$
H^{q}\left(N_{ \pm}, R\right)= \begin{cases}R & \text { for } q \equiv 0(\bmod \mu), 0<q<n \\ R & \text { for } q \equiv m_{\mp 1}(\bmod \mu), 0<q<n \\ 0 & \text { otherwise. }\end{cases}
$$

Furthermore,

$$
H^{q}(N, R)= \begin{cases}R & \text { for } q=0 \text { or } n \\ H^{q}\left(N_{+}\right) \oplus H^{q}\left(N_{-}\right) & \text {for } 1 \leq q \leq n-1 .\end{cases}
$$

\subsection{Gauss images of isoparametric hypersurfaces}

We shall consider an isoparametric hypersurface $N$ in $S^{n+1}$ as an oriented hypersurface, and consider its Gauss map

$$
\mathcal{G}: N \ni p \longmapsto[\boldsymbol{x}(p)+\sqrt{-1} \boldsymbol{n}(p)] \in \widetilde{\mathrm{Gr}}_{2}\left(\mathbb{R}^{n+2}\right) \cong Q_{n}(\mathbb{C}) .
$$

Proposition 2.5 ([34], [19], [29]). Suppose that $N$ is an isoparametric hypersurface of $S^{n+1}(1)$. Then the following properties hold:

(1) The Gauss map of $N$

$$
\mathcal{G}: N \longrightarrow \widetilde{\mathrm{Gr}}_{2}\left(\mathbb{R}^{n+2}\right) \cong Q_{n}(\mathbb{C})
$$

is a minimal Lagrangian immersion into $Q_{n}(\mathbb{C})$.

(2) Moreover the Gauss image $L=\mathcal{G}(N)$ is a compact minimal Lagrangian submanifold embedded in $Q_{n}(\mathbb{C})$ and the Gauss map gives a covering map onto the Gauss image

$$
\mathcal{G}: N \longmapsto L=\mathcal{G}(N) \cong N / \mathbb{Z}_{\mathrm{g}}
$$

with the covering transformation group $\mathbb{Z}_{g}$.

(3) $N^{n} \subset S^{n+1}$ is homogeneous if and only if the Gauss image $L=\mathcal{G}\left(N^{n}\right)$ is homogeneous.

(4) $\frac{2 n}{g}$ is even (resp.odd) if and only if its Gauss image $L=\mathcal{G}\left(N^{n}\right)$ is orientable (resp. non-orientable).

Some problems such as classification of homogeneous Lagrangian submanifolds, Hamiltonian stability and so on were studied in [19], [20], [21], [22] for the Gauss images of isoparametric hypersurfaces.

Here we refer

Proposition 2.6 ([8], [32]). Let $(M, \omega, J, g)$ be an Einstein-Kähler manifold of Einstein constant $>0$. Then any compact minimal Lagrangian submanifold $L$ of $M$ is monotone.

The definition of the monotonicity for Lagrangian submanifolds is given in Section 5. By this result we see that the Gauss image of an isoparametric hypersurface is always a monotone Lagrangian submanifold of $Q_{n}(\mathbb{C})$.

Now we discuss the intersection theory of those minimal Lagrangian submanifolds $L=\mathcal{G}(N)$ in $Q_{n}(\mathbb{C})$.

\section{A review of symplectic geometry}

Let $\left(M^{2 n}, \omega\right)$ be a symplectic manifold, namely, $\omega$ is a non-degenerate closed 2-form on $M$. Let $\iota: L \rightarrow M$ be a Lagrangian submanifold, namely, $\operatorname{dim} L=n$ and $\iota^{\star} \omega=0$.

Example 3.1 :

(1) For any manifolds $X$, the cotangent vector bundle $T^{\star} X$ is a symplectic manifold with canonical coordinate $\operatorname{system}(x, \xi)$, and $\omega^{T^{*} M}=\sum d x^{i} \wedge d \xi^{i}$. The zero section $L=X$ and each fiber $L=\pi^{-1}(x)(x \in X)$ both are Lagrangian submanifolds of $T^{\star} X$. 
(2) Any Kähler manifold and so any oriented Riemannian surface are symplectic manifolds. On such surfaces, any curve is a Lagrangian submanifold.

\subsection{Weinstein neighborhood}

A Lagrangian submanifold $L$ in $(M, \omega)$ is also a Lagrangian submanifold of $T^{\star} L$ as the zero section at the same time. It is well-known that there exist a tubular neighborhood $\left(W(L),\left.\omega\right|_{W(L)}\right)$ of $L$ in $M$ and a tubular neighborhood $\left(U\left(0_{L}\right),\left.\omega^{T^{*} L}\right|_{U\left(0_{L}\right)}\right)$ of the zero section $0_{L}$ in $T^{\star} L$, which are symplectomorphic to each other.

Definition. We call $W(L)$ a Weinstein neighborhood of $L$ in $M$.

\subsection{Hamiltonian diffeomorphisms}

In the following, let $(M, \omega)$ be a closed (i.e. compact without boundary) symplectic manifold.

\section{Definition.}

(1) $\left\{\phi_{t}^{H}\right\}_{t \in[0,1]}$ is called a Hamiltonian isotopy of $M$, when for a time dependent Hamiltonian function $H$ : $[0,1] \times M \rightarrow \mathbb{R}, \phi_{t}^{H}$ is a Hamiltonian flow associated with $X_{H_{t}}$,

$$
\frac{d \phi_{t}^{H}(p)}{d t}=\left(X_{H_{t}}\right)_{p}, \phi_{0}^{H}(p)=p \quad(\forall p \in M)
$$

where $X_{H_{t}}$ is the Hamiltonian vector field corresponding to $H_{t}$ defined by $d H_{t}=\omega\left(, X_{H_{t}}\right)$.

(2) The time 1 map $\varphi=\phi_{1}^{H}$ is called a Hamiltonian diffeomorphism of $M$.

Denote the Hamiltonian diffeomorphism group of $(M, \omega)$ by

$$
\begin{aligned}
\operatorname{Ham}(M, \omega) & :=\{\text { Hamiltonian diffeomorphisms of } M\} \\
\subset \operatorname{Sym}^{0}(M, \omega) & =\{\text { symplectomorphisms isotopic to the identity map }\} .
\end{aligned}
$$

Sometimes we call $\varphi \in \operatorname{Ham}(M, \omega)$ a Hamiltonian isotopy or a Hamiltonian deformation. Any $\varphi \in \operatorname{Ham}(M, \omega)$ maps Lagrangian submanifolds to Lagrangian submanifolds.

$$
\begin{aligned}
& \text { Question. If } L \text { is a closed embedded Lagrangian submanifold of } M, \\
& \quad \text { does it hold the inequality } \\
& \left.\#(L \cap \varphi(L)) \geq \mathrm{SB}\left(L, \mathbb{Z}_{2}\right) \text { (=sum of the Betti numbers of } L\right) \text { ? }
\end{aligned}
$$

In general, this FAILS as a large isometry $\varphi$ for a small circle $L$ in $S^{2}$ gives a counter-example. On the other hand, a great circle of $S^{2}$ satisfies it. Therefore, the Lagrangian intersection is considered as not only a topological matter, but also a symplectic matter.

\subsection{Lagrangian graph}

A simple case: For any manifold $L^{n}$ and $f \in C^{\infty}(L), L_{f}=\left\{\left(x,(d f)_{x}\right)\right\} \subset T^{\star} L$ is a Lagrangian submanifold, because $d f=f_{i} d x^{i}$ and so $\omega^{T^{*} L_{L_{f}}}=\sum d x^{i} \wedge d f_{i}=\sum d x^{i} \wedge f_{i j} d x^{j}=0$.

Definition. $L_{f}$ is called a Lagrangian graph in $T^{\star} L$.

Since $L \cap L_{f}=\{(x, 0)\} \cap L_{f}$ is nothing but the critical point set of $f$, when $L$ is closed and $f$ is a Morse function, the Morse inequality implies the Arnold inequality

$$
\sharp\left(L \cap L_{f}\right) \geq S B\left(L, \mathbb{Z}_{2}\right) .
$$

Let $L$ be a Lagrangian submanifold of a symplectic manifold $(M, \omega)$. When $\varphi \in \operatorname{Ham}(M, \omega)$ is small, $\varphi(L) \subset W(L)$ where $W(L)$ is the Weinstein neighborhood. However in general $\varphi(L)$ outgrows $W(L)$. 
Now the Floer theory plays an important role. This is a Morse theory on infinite dimensional spaces. Let us briefly review the Morse theory on finite dimensional manifolds.

Let $M$ be a closed manifold and $f \in C^{\infty}(M)$ be a Morse-Smale function. Let

$$
\operatorname{Crit}(f):=\{\text { critical points of } f\}
$$

and

$$
\operatorname{Crit}_{k}(f):=\{\text { critical points of } f \text { of index } k\} .
$$

Define the Morse complex by a vector space over $\mathbb{Z}_{2}$ with a grading

$$
C_{\star}^{f}:=\bigoplus_{k=0}^{n} C_{k}^{f}, \quad \text { where } \quad C_{k}^{f}:=\bigoplus_{x \in \operatorname{Crit}_{k}(f)} \mathbb{Z}_{2} x .
$$

For $x, y \in \operatorname{Crit}(f)=\bigcup_{k=0}^{n} \operatorname{Crit}_{k}(f)$, define

$$
\begin{aligned}
& \mathcal{M}(x, y):= \\
& \left\{\gamma(s): \mathbb{R} \rightarrow M \mid \frac{d \gamma}{d s}=-\operatorname{grad} f, \lim _{s \rightarrow-\infty} \gamma(s)=x, \lim _{s \rightarrow \infty} \gamma(s)=y\right\} / \sim,
\end{aligned}
$$

where $\sim$ is a parameter shift $s \mapsto s+a$.

The Morse boundary operator $\partial^{f}: C_{k}^{f} \rightarrow C_{k-1}^{f}$ is defined by

$$
\partial^{f} x=\sum_{y \in C_{k-1}} \sharp \mathcal{N}(x, y) y,
$$

for each $x \in C_{k}^{f}$, where the number of elements $\sharp \mathcal{M}(x, y)$ is counted modulo 2. Then $\partial^{f} \circ \partial^{f}=0$, and so the Morse homology $H\left(C_{\star}^{f}, \partial^{f}\right)=\frac{\operatorname{Ker} \partial^{f}}{\operatorname{Im} \partial^{f}}$ is defined and it is isomorphic to $H\left(M, \mathbb{Z}_{2}\right)$. The Morse homology over the coefficient $\mathbb{Z}_{2}$ is elementary. The Morse homology is usually constructed over the coefficient $\mathbb{Z}$, under the setup together with the orientations.

\section{Floer homology of Lagrangian intersection}

Let $L \subset(M, \omega)$ be a closed Lagrangian submanifold, and $\varphi=\phi_{1} \in \operatorname{Ham}(M, \omega)$. We suppose that

$$
[C] \quad \int_{D} v^{*} \omega=0 \quad \text { for all smooth } v:\left(D^{2}, \partial D^{2}\right) \rightarrow(M, L) .
$$

Fix a base point $x_{0} \in L$. Set

$$
\Omega:=\left\{z:[0,1] \rightarrow M \mid z(0) \in L, z(1) \in \varphi(L), \text { isotopic to } \phi_{t}\left(x_{0}\right)\right\} .
$$

and define a 1-form on $\Omega$ as

$$
\alpha_{z}(\xi):=\int_{0}^{1} \omega_{z(t)}(\dot{z}(t), \xi(t)) d t
$$

Then we observe that $\alpha$ is closed.

Theorem 4.1 ([12]). Under the condition [C], the following hold:

(1) There exists a functional $\mathcal{A}$ locally defined on $\Omega$ such that $\alpha=d \mathcal{A}$.

(2) $z \in \Omega$ is a critical point of $\mathcal{A}$ if and only if $\dot{z}(t)=0$, namely, $z$ is a constant path $z(t)=x \in L \cap \varphi(L)$. Moreover, a critical point $z$ of $\mathcal{A}$ is non-degenerate if and only if $L$ and $\varphi(L)$ transversally intersect at $z$, 
(3) For $J=\left\{J_{t}\right\}_{0 \leq t \leq 1}$, a time-dependent family of almost complex structures on $M$ compatible with $\omega$, we have

$$
(\operatorname{grad} \mathcal{A})_{z}(t)=\left(J_{t}\right)_{z(t)} \dot{z}(t) \quad(t \in[0,1]) .
$$

Definition. For $x, y \in L \cap \varphi(L)$, define the moduli space

$$
\begin{aligned}
& \mathcal{M}(x, y):= \\
& \left\{u: \mathbb{R} \times[0,1] \rightarrow M \mid \frac{\partial u}{\partial s}=-\operatorname{grad} \mathcal{A}, \lim _{s \rightarrow-\infty} u=x, \lim _{s \rightarrow \infty} u=y\right\} / \sim
\end{aligned}
$$

Each element $u \in \mathcal{M}(x, y)$ is called a J-holomorphic strip. In fact, if we put $u(s, t)=z_{s}(t)$, then it satisfies a kind of Cauchy-Riemann equation

$$
\frac{\partial u(s, t)}{\partial s}+J_{t} \frac{\partial u(s, t)}{\partial t}=0 .
$$

Suppose that the intersection $L \cap \varphi(L)$ is transversal. Then the following properties of the moduli space $\mathcal{M}(x, y)$ hold:

Theorem 4.2 ([37], [12]). Under the condition [C], the following hold:

(1) For a generic $J=\left\{J_{t}\right\}_{0 \leq t \leq 1}$, the Maslov-Viterbo index $\mu_{u}(x, y) \in \mathbb{Z}$ is defined for each $x, y \in L \cap \varphi(L)$ and each $u \in \mathcal{M}(x, y)$, so that a neighborhood around $u$ in the moduli space $\mathcal{N}(x, y)$ is a $\left(\mu_{u}(x, y)-1\right)$ dimensional smooth manifold.

(2) The 0-dimensional component $\mathcal{M}^{0}(x, y)$ of $\mathcal{M}(x, y)$ is compact.

(3) The boundary $\partial \overline{\mathcal{M}^{1}(x, y)}$ of the 1-dimensional component $\mathcal{M}^{1}(x, y)$ of $\mathcal{M}(x, y)$ is given by

$$
\bigcup_{z \in L \cap \varphi(L)} \mathcal{M}^{0}(x, z) \times \mathcal{M}^{0}(z, y)
$$

Define the Floer complex by a vector space over $\mathbb{Z}_{2}$

$$
C F(L, \varphi):=\bigoplus_{p \in L \cap \varphi(L)} \mathbb{Z}_{2} p,
$$

and the Floer boundary operator $\partial_{J}: C F(L, \varphi) \rightarrow C F(L, \varphi)$ by

$$
\partial_{J} x=\sum_{y \in L \cap \Phi(L)} \sharp \mathcal{N}^{0}(x, y) y,
$$

where the number of elements $\sharp \mathcal{M}^{0}(x, y)$ is counted modulo 2. Combining this with (3), we obtain $\partial_{J} \circ \partial_{J}=0$, and the Floer homology is a vector space over $\mathbb{Z}_{2}$ defined by

$$
H F(L):=\frac{\operatorname{Ker} \partial_{J}}{\operatorname{Im} \partial_{J}} .
$$

By definition it obviously holds the inequality

$$
\#(L \cap \varphi(L)) \geq \operatorname{rank}_{\mathbb{Z}_{2}} H F(L) .
$$

Choose a base intersection point $x_{0} \in L \cap \varphi(L)$. Using the Maslov-Viterbo index $\mu_{u}\left(x, x_{0}\right)$, and minimal Maslov number $\Sigma_{L}$ (see the next section for the definition), one can define a $\mathbb{Z} / \Sigma_{L}$-grading on the Floer complex $C F$ as

$$
C F(L, \varphi)=\bigoplus_{i=0}^{\Sigma_{L}-1} C F_{i \bmod \Sigma_{L}}\left(L, \varphi, x_{0}\right) .
$$

and then $\partial_{J}: C F_{i \bmod \Sigma_{L}}\left(L, \varphi, x_{0}\right) \rightarrow C F_{i-1 \bmod \Sigma_{L}}\left(L, \varphi, x_{0}\right)$. Hence we have a $\mathbb{Z} / \Sigma_{L}$-grading on the Floer homology

$$
H F(L)=\bigoplus_{i=0}^{\Sigma_{L}-1} H F_{i \bmod \Sigma_{L}}\left(L, \varphi, x_{0}\right) .
$$


Theorem 4.3 ([12]). Under the condition [C], the following hold:

(1) $H F(L)$ is well-defined and independent of the choice of $H_{t}$ and generic $J_{t}$. In particular, $H F(L)$ is invariant under any Hamiltonian diffeomorphism and its $\mathbb{Z} / \Sigma_{L}$-grading is also preserved under any Hamiltonian diffeomorphism.

(2) If $\pi_{2}(M, L)=0$, then $H F(L) \cong H_{\star}\left(L, \mathbb{Z}_{2}\right)$.

\section{Generalization of Floer homology by Y.G.Oh}

Y.G. Oh weakened the condition [C] to monotone Lagrangian submanifolds, and showed that all results in Section 4 except for Theorem 4.3 (2) hold.

Definition. A group homomorphism $I_{\omega}: \pi_{2}(M, L) \rightarrow \mathbb{R}$ is defined by

$$
I_{\omega}(A)=\int_{D} u^{\star} \omega
$$

for each smooth map $u:(D, \partial D) \rightarrow(M, L)$ with $[u]=A \in \pi_{2}(M, L)$.

On the other hand, another group homomorphism $I_{\mu, L}: \pi_{2}(M, L) \rightarrow \mathbb{Z}$ is defined by

$$
I_{\mu, L}(A)=\mu(\tilde{u}),
$$

where set $\tilde{u}:=\left.u\right|_{\partial D}: S^{1} \rightarrow \Lambda\left(\mathbb{C}^{n}\right), \Lambda\left(\mathbb{C}^{n}\right)$ is the Lagrangian Grassmann manifold consisting of all Lagrangian vector subspaces of $\mathbb{C}^{n}$, and $\mu \in H^{1}\left(\Lambda\left(\mathbb{C}^{n}\right), \mathbb{Z}\right) \cong \mathbb{Z}$ is the Maslov class of $\Lambda\left(\mathbb{C}^{n}\right)$.

Definition. The positive generator $\Sigma_{L}$ of the image of $I_{\mu, L}$ is called the minimal Maslov number of $L$. When $I_{\mu, L}=0$, we define $\Sigma_{L}=0$.

Definition. $L$ is said to be monotone if there exists $\lambda>0$ such that

$$
I_{\mu, L}=\lambda I_{\omega}
$$

Theorem 5.1 ([26], [27], [28]). When $L$ is monotone and has minimal Maslov number $\Sigma_{L} \geq 2$, the homology $H F(L):=H_{\star}\left(C F(L), \partial_{J}\right)$ is well-defined for a generic choice of $(H, J)$. This is called the Floer homology of $L$ with $\mathbb{Z}_{2}$-coefficient, and this is invariant under the Hamiltonian isotopies of $L$.

Suppose that $L$ is monotone and $\Sigma_{L} \geq 2$. We consider a Morse-Smale function $f$ on $L$ so that a Hamiltonian isotopy $\phi_{t}(L)=(d(t f))_{L}(t \in[0,1])$ is contained in a Weinstein neighborhood of $L$ identified with a tubular neighborhood of the zero section of $T^{\star} L$. In such a setting, $L \cap \phi_{1}(L)$ coincides with Crit $(f)$ (see Subsection 3.3). We may assume that a base intersection point $x_{0}$ is a unique relative minimum point of $f$ on $L$. Then we know that the Maslov-Viterbo index $\mu_{u}\left(x, x_{0}\right)$ coincide with the Morse index of $f$ at a critical point $x$ and we have

$$
C F_{i \bmod \Sigma_{L}}\left(L, \phi_{1}, x_{0}\right)=\bigoplus_{k \in \mathbb{Z}, k \equiv i \bmod \Sigma_{L}} C_{k}^{f} .
$$

Set $C F_{k}:=C_{k}^{f}$. Moreover the Floer boundary operator can be decomposed as

$$
\partial_{J}=\partial_{0}+\partial_{1}+\cdots+\partial_{v}, \quad \partial_{l}: C_{\star}^{f} \rightarrow C_{\star_{-}+l+l \Sigma_{L}}^{f},
$$

where $\partial_{0}$ is the Morse boundary operator and the other $\partial_{j}(j=1, \cdots, v)$ makes indices jump, where $v:=\left[\frac{\operatorname{dim} L+1}{\Sigma_{L}}\right]$. By this decomposition of the Floer boundary operator, the spectral sequence for the Floer homology were constructed by $\mathrm{Oh}$ [28] and Biran [2]. The calculation of $H F(L)$ is easier if $v$ is small, but more difficult if $v$ is large.

Remark 5.2 : When $\Sigma_{L}=2$, the bubbling of $J$-holomorphic strips may occur, and the calculation becomes difficult. 
The Gauss image $L=\mathcal{G}(N)$ of an isoparametric hypersurface $N$ is a monotone Lagrangian submanifold in $Q_{n}(\mathbb{C})$, and so we can apply Oh's argument to it.

\section{Definition.}

(1) When there exists $\varphi \in \operatorname{Ham}(M, \omega)$ such that $L \cap \varphi(L)=\emptyset$, we say $L$ is Hamiltonian displaceable.

(2) When $L \cap \varphi(L) \neq \emptyset$ for any $\varphi \in \operatorname{Ham}(M, \omega)$, we say $L$ is Hamiltonian non-displaceable.

Since the Floer homology is generated by intersection points, it is obvious that if $L \subset(M, \omega)$ is Hamiltonian displaceable, then we have $H F(L)=0$.

\section{Results}

Let $N$ be an isoparametric hypersurface with $g$ distinct principal curvatures, and set $L=\mathcal{G}(N) \subset Q_{n}(\mathbb{C})$ as its Gauss image. First the following proposition is known and elementary in submanifold theory:

Proposition 6.1. $g=1$ or 2 if and only if $L=\mathcal{G}(N)$ is a real form, (equivalently, a totally geodesic Lagrangian submanifold) of $Q_{n}(\mathbb{C})$.

(1) $g=1\left(N=S^{n}\right) \Rightarrow L=S^{n} \subset Q_{n}(\mathbb{C})$ is a real form of $Q_{n}(\mathbb{C})$.

(2) $g=2\left(N=S^{k} \times S^{n-k}, 1 \leq k \leq n-1\right) \Rightarrow L=\left(S^{k} \times S^{n-k}\right) / \mathbb{Z}_{2} \subset Q_{n}(\mathbb{C})(1 \leq k \leq n-1)$ are real forms of $Q_{n}(\mathbb{C})$.

Proposition 6.2 ([28], [18]). In these cases, $H F(L) \cong H_{*}\left(L, \mathbb{Z}_{2}\right) \neq 0$ and thus $L$ is Hamiltonian nondisplaceable.

Question. How about the case $\mathrm{g}>2$ ?

As for the minimal Maslov number $\Sigma_{L}$ of the Gauss image $L=\mathcal{G}(N)$ of an isoparametric hypersurface, it holds

Lemma 6.3 ([21], [29]).

$$
\Sigma_{L}=\frac{2 n}{g}= \begin{cases}m_{1}+m_{2} & \text { if } g \text { is even, } \\ 2 m & \text { if } g \text { is odd, }\end{cases}
$$

(see Theorem 2.2(1)).

Now for $g>2$, we state our main result.

\section{Main Theorem ([17]).}

(1) $g=3 \Rightarrow L=\mathcal{G}(N)$ is a $\mathbb{Z}_{2}$-homology sphere.

If $m=m_{i} \geq 2, \Rightarrow H F(L) \cong H_{\star}\left(L, \mathbb{Z}_{2}\right)$, and in particular,

$L$ is Hamiltonian non-displaceable, and

$$
\#(L \cap \varphi(L)) \geq S B\left(L, \mathbb{Z}_{2}\right)=2 .
$$

(2) $g=4$ and $2 \leq m_{1} \leq m_{2} \Rightarrow L$ is Hamiltonian non-displaceable.

(3) $g=6$ and $m=m_{i}=2 \Rightarrow L$ is Hamiltonian non-displaceable.

Remark 6.4 : We need $\Sigma_{L} \geq 3$ for the proof, where $\Sigma_{L}=m_{1}+m_{2}$ by Lemma 6.3. Since $m_{1}=m_{2}$ for $g=3,6$, $m=m_{i} \geq 2$ is necessary in (1) and (3). When $\left(g, n, m_{1}, m_{2}\right)=(4,2(1+k), 1, k)(k \geq 2), \Sigma_{L}=k+1 \geq 3$ holds. 
However, when we apply Damian's spectral sequence in the proof, we need $m_{1} \geq 2$ (see below). Thus the remaining cases are

$$
\begin{array}{rlrl}
\left(g, n, m_{1}, m_{2}\right)= & (3,3,1,1), & N=\frac{S O(3)}{\mathbb{Z}_{2}+\mathbb{Z}_{2}}, \\
\left(g, n, m_{1}, m_{2}\right)= & (4,2 k+2,1, k), & N=\frac{S O(2) \times S O(k+2)}{\mathbb{Z}_{2} \times S O(k)}, \\
& \left(k \geq 1, \Sigma_{L}=k+1\right), & & \\
\left(g, n, m_{1}, m_{2}\right)= & (6,6,1,1), & N=\frac{S O(4)}{\mathbb{Z}_{2}+\mathbb{Z}_{2}} .
\end{array}
$$

It is known that all isoparametric hypersurfaces of such $\left(g, n, m_{1} . m_{2}\right)$ are homogeneous.

\section{Strategy of the proof}

As for (1), we directly compute $\pi_{k}(L)$ to show that $L$ is a $\mathbb{Z}_{2}$-homology sphere, see [17], Lemma 4.1.

Here, we will give the strategy of the proof for (2) and (3).

Because the Gauss map $\mathcal{G}: N \rightarrow Q_{n}(\mathbb{C})$ is a covering map onto $N / \mathbb{Z}_{g}=L$, we use Damian's lifted Floer homology $H F^{\bar{L}}(L)$ for a covering map $\bar{L}=N \rightarrow L$. This is shown to be invariant under Hamiltonian isotopies of $L$. We give more details to show

$H F^{N}(L) \neq 0$ and so $L \cap \varphi(L) \neq \emptyset, L$ is Hamiltonian non-displaceable.

Let $(M, \omega)$ be a closed symplectic manifold and $L \subset M$ be a closed embedded monotone Lagrangian submanifold with $\Sigma_{L} \geq 3$. Let us regard the intersection $C=L \cap \varphi(L)$ as points in $L$ where $\varphi$ is a Hamiltonian diffeomorphism. For any two points $x, y \in C$, consider an isolated $J$-holomorphic strip $v: R \times[0,1] \rightarrow M$ from $x$ to $y$, which defines a path $\gamma$ in $L$ from $x$ to $y$ by $\gamma(s):=v(s, 0)$. We denote by $\Gamma$ all the paths $\gamma$ given by this procedure. Note that to reconstruct the Floer complex $\left(C F(L), \partial_{J}\right)$, it is enough to know the above collection $(C, \Gamma)$ of points and paths.

Starting with the data $(C, \Gamma)$, fix an arbitrary covering $\pi: \tilde{L} \rightarrow L$. For any point $x \in C$, denote by $x_{i}, i \in I$ the elements of the fiber $\pi^{-1}(x)$. Consider the set $\tilde{\Gamma}$ of all the lifts of the paths of $\Gamma$ to the covering space $\tilde{L}$. For any points $x_{i}, y_{j}(i, j \in I)$, the cardinality of elements in $\Gamma$ that connect $x_{i}$ with $y_{j}$ is finite. We denote by $n\left(x_{i}, y_{j}\right)$ its parity. Let $C F^{\tilde{L}}(L)$ be the free $\mathbb{Z}_{2}$-module generated by $\cup_{x \in C} \pi^{-1}(x)$. One can define the boundary operator $\partial^{\tilde{L}}$ on $C F^{\tilde{L}}(L)$ by the formula

$$
\partial^{\tilde{L}}\left(x_{i}\right)=\sum_{\pi\left(y_{i}\right)=y \in C} n\left(x_{i}, y_{j}\right) y_{j} .
$$

Damian ([9], Proposition 2.6) proved that $\left(C F^{\tilde{L}}(L), \partial^{\tilde{L}}\right)$ is a chain complex and its homology $H F^{\tilde{L}}(L):=$ $H *\left(C F^{\tilde{L}}(L), \partial^{\tilde{L}}\right)$ is called the lifted Floer homology of $L$. Moreover, this is invariant under the Hamiltonian isotopies of $L$.

If $L$ is Hamiltonian displaceable, the Floer complex $C=L \cap \varphi(L)$ is empty for some Hamiltonian diffeomorphism $\varphi$, and so is the lifted Floer complex, which implies $H F^{\tilde{L}}(L)=0$ (see also Remark 7.3 below).

Next, we use Damian's spectral sequence as follows:

For $\Lambda=\mathbb{Z}_{2}\left[T, T^{-1}\right]$, let $\Lambda^{i} \subset \Lambda$ be the subspace of homogeneous elements of degree $i$.

Proposition 7.1 ([9]). For $p, q \in \mathbb{Z}$, there exists a spectral sequence $\left\{E_{r}^{p, q}, d_{r}\right\}$ satisfying the following properties:

1. $E_{0}^{p, q}=C F_{p+q-p \Sigma_{L}}^{\bar{L}} \otimes \Lambda^{p \Sigma_{L}}, d_{0}=\left[\partial_{0}^{\bar{L}}\right] \otimes 1$.

2. $E_{1}^{p, q}=H_{p+q-p \Sigma_{L}}\left(\bar{L}, \mathbb{Z}_{2}\right) \otimes \Lambda^{p \Sigma_{L}}, d_{1}=\left[\partial_{1}^{\bar{L}}\right] \otimes T^{-\Sigma_{L}}$, where

$$
\left[\partial_{1}^{\bar{L}}\right]: H_{p+q-p \Sigma_{L}}\left(\bar{L} ; \mathbb{Z}_{2}\right) \rightarrow H_{p+q-1-(p-1) \Sigma_{L}}\left(\bar{L} ; \mathbb{Z}_{2}\right)
$$


is induced by $\partial_{1}^{\bar{L}}$.

3. For any $r \geq 1, E_{r}^{p, q}=V_{r}^{p, q} \otimes \Lambda^{p \Sigma_{L}}$ with $d_{r}=\delta_{r} \otimes T^{-r \Sigma_{L}}$, where $V_{r}^{p, q}$ is a vector space over $\mathbb{Z}_{2}$ and $\delta_{r}: V_{r}^{p, q} \rightarrow$ $V_{r}^{p-r, q+r-1}$ is a homomorphism defined for every $p$, $q$ and satisfies $\delta_{r} \circ \delta_{r}=0$. More precisely,

$$
\begin{gathered}
V_{r+1}^{p, q}=\frac{\operatorname{Ker}\left(\delta_{r}: V_{r}^{p, q} \rightarrow V_{r}^{p-r, q+r-1}\right)}{\operatorname{Im}\left(\delta_{r}: V_{r}^{p+r, q-r+1} \rightarrow V_{r}^{p, q}\right)} \\
V_{0}^{p, q}=C F_{p+q-p \Sigma_{L}}^{\bar{L}}, \quad V_{1}^{p, q}=H_{p+q-p \Sigma_{L}}\left(\bar{L}_{2} ; \mathbb{Z}_{2}\right), \delta_{1}=\left[\partial_{1}^{\bar{L}}\right]
\end{gathered}
$$

4. $E_{r}^{p, q}$ collapses at $(v+1)$-step and for any $p \in \mathbb{Z}, \oplus_{q \in \mathbb{Z}} E_{\infty}^{p, q} \cong H F^{\bar{L}}(L)$, where $v=\left[\frac{\operatorname{dim} L+1}{\Sigma_{L}}\right]$.

Back to the Gauss image, $v=\left[\frac{\operatorname{dim} L+1}{\Sigma_{L}}\right]=\left[\frac{(n+1) g}{2 n}\right]$ implies:

Lemma 7.2. For a Gauss image $L^{n}=\mathcal{G}\left(N^{n}\right) \subset Q_{n}(\mathbb{C}), g \geq 3$ and any $p, q \in \mathbb{Z}$, we have

1. $E_{2}^{p, q}=E_{\infty}^{p, q}$ if and only if $g=3$ and $\left(m_{1}, m_{2}\right)=(2,2),(4,4),(8,8)$.

2. $E_{3}^{p, q}=E_{\infty}^{p, q}$ if and only if $g=3,\left(m_{1}, m_{2}\right)=(1,1)$ or $g=4$.

3. $E_{4}^{p, q}=E_{\infty}^{p, q}$ if and only if $g=6,\left(m_{1}, m_{2}\right)=(1,1)$ or $(2,2)$.

Using these, we give a sketch of the proof for $g=4$. The case $g=6$ is similarly obtained although a bit longer.

When $g=4$, suppose $L$ is Hamiltonian displaceable, hence $H F^{\bar{L}}(L)=0$. Then by Lemma 7.2 (2) we have $0=E_{3}^{0, q}$ and so it follows from Proposition 7.1 (3) that

$$
V_{2}^{2, q-1} \rightarrow V_{2}^{0, q} \rightarrow V_{2}^{-2, q+1} \quad \text { is exact. }
$$

Since

$$
\begin{aligned}
& V_{2}^{2, q-1}= \frac{\operatorname{Ker}\left(\left[\partial_{1}^{\bar{L}}\right]: H_{q+1-2 \Sigma_{L}}\left(\bar{L} ; \mathbb{Z}_{2}\right) \rightarrow H_{q-\Sigma_{L}}\left(\bar{L} ; \mathbb{Z}_{2}\right)\right)}{\operatorname{Im}\left(\left[\partial_{1}^{\bar{L}}\right]: H_{q+2-3 \Sigma_{L}}\left(\bar{L} ; \mathbb{Z}_{2}\right) \rightarrow H_{q+1-2 \Sigma_{L}}\left(\bar{L} ; \mathbb{Z}_{2}\right)\right)}, \\
& V_{2}^{-2, q+1}=\frac{\operatorname{Ker}\left(\left[\partial_{1}^{\bar{L}}\right]: H_{q-1+2 \Sigma_{L}}\left(\bar{L} ; \mathbb{Z}_{2}\right) \rightarrow H_{q-2+3 \Sigma_{L}}\left(\bar{L} ; \mathbb{Z}_{2}\right)\right)}{\operatorname{Im}\left(\left[\partial_{1}^{\bar{L}}\right]: H_{q+\Sigma_{L}}\left(\bar{L} ; \mathbb{Z}_{2}\right) \rightarrow H_{q-1+2 \Sigma_{L}}\left(\bar{L} ; \mathbb{Z}_{2}\right)\right)},
\end{aligned}
$$

$V_{2}^{2, q-1}=V_{2}^{-2, q+1}=0$ when $2 \leq q \leq n-2$.

Then it follows

$$
0=V^{0, q}=\frac{\operatorname{Ker}\left(\left[\partial_{1}^{\bar{L}}\right]: H_{q}\left(\bar{L} ; \mathbb{Z}_{2}\right) \rightarrow H_{q-1+\Sigma_{L}}\left(\bar{L} ; \mathbb{Z}_{2}\right)\right)}{\operatorname{Im}\left(\left[\partial_{1}^{\bar{L}}\right]: H_{q+1-\Sigma_{L}}\left(\bar{L} ; \mathbb{Z}_{2}\right) \rightarrow H_{q}\left(\bar{L} ; \mathbb{Z}_{2}\right)\right)}
$$

for $2 \leq q \leq n-2$. Putting $q=\Sigma_{L}=m_{1}+m_{2}$, we know

$$
H_{1}\left(\bar{L} ; \mathbb{Z}_{2}\right) \rightarrow H_{m_{1}+m_{2}}\left(\bar{L} ; \mathbb{Z}_{2}\right) \rightarrow H_{2\left(m_{1}+m_{2}\right)-1}\left(\bar{L} ; \mathbb{Z}_{2}\right)
$$

is exact.

On the other hand, by Münzner's Theorem 2.4, we know

$$
H_{k}\left(N ; \mathbb{Z}_{2}\right) \cong \begin{cases}\mathbb{Z}_{2}, & \text { for } k=0, m_{1}, m_{2}, 2 m_{1}+m_{2}, m_{1}+2 m_{2}, n, \\ \mathbb{Z}_{2} \oplus \mathbb{Z}_{2}, & \text { for } k=m_{1}+m_{2}, \\ 0, & \text { otherwise. }\end{cases}
$$

Thus this contradits (1) if $2 \leq m_{1} \leq m_{2}$.

As a result, $L$ is Hamiltonian non-displaceable when $g=4$ and $2 \leq m_{1} \leq m_{2}$.

Remark 7.3 : Note that in general, $H F^{\bar{L}}(L) \neq 0$ does not imply $H F(L) \neq 0$ and it is an open problem to determine whether $H F(L) \neq 0$ or not in our case. 
We shall mention some results related to Main Theorem. It is also interesting to study extrinsic topology of the Gauss images of isoparametric hypersurfaces in complex hyperquadrics. The following result of Albers gives a sufficient condition on extrinsic topology for monotone Lagrangian submanifolds to be Hamiltonian non-displaceable.

Theorem $7.4([1])$. Let $\left(M^{2 n}, \omega\right)$ be a monotone closed symplectic manifold. Let $L$ be a monotone compact Lagrangian submanifold of $M$ with minimal Maslov number $\Sigma_{L} \geq 3$. If $L$ is Hamiltonian displaceable, then the induced homology homomorphism $\iota *: H_{k}\left(L ; \mathbb{Z}_{2}\right) \rightarrow H_{k}\left(M ; \mathbb{Z}_{2}\right)$ vanishes for degrees $k>\operatorname{dim} L+1-\Sigma_{L}$ and in particular $\iota_{*}[L]=0$ in $H_{n}\left(M ; \mathbb{Z}_{2}\right)$.

However it does not give any new information in our cases, as we observed in [31, Propositions 4.6, 4.8].

Proposition 7.5 ([31]). (1) Assume that $M=Q_{n}(\mathbb{C})$ and $L^{n}=\mathcal{G}\left(N^{n}\right)$ is the Gauss image of an isoparametric hypersurface $N^{n}$ except for the case when $g=1$ and $n \geq 2$. Then the induced $\mathbb{Z}$-homology homomorphism

$$
\iota \star H_{k}(L ; \mathbb{Z}) \longrightarrow H_{k}(M ; \mathbb{Z})
$$

and the induced $\mathbb{Z}_{2}$-homology homomorphism

$$
\iota \star H_{k}\left(L ; \mathbb{Z}_{2}\right) \longrightarrow H_{k}\left(M ; \mathbb{Z}_{2}\right)
$$

vanish for degrees $\operatorname{dim} L>k>\operatorname{dim} L+1-\Sigma_{L}$. If $g=1$ and $n \geq 2$, then for $k=0>1-n=\operatorname{dim} L+1-\Sigma_{L}$, the corresponding homology homomorphisms do not vanish (and thus $L$ is Hamiltonian non-displaceable).

(2) The induced $\mathbb{Z}_{2}$-homology homomorphism

$$
\iota \star: H_{n}\left(L^{n} ; \mathbb{Z}_{2}\right) \longrightarrow H_{n}\left(Q_{n}(\mathbb{C}) ; \mathbb{Z}_{2}\right)
$$

vanishes if $L^{n}=\mathcal{G}\left(N^{n}\right)$ is the Gauss image of an isoparametric hypersurface $N^{n}$ in the following list:

$$
\begin{array}{ll}
g=1, & n \text { is odd. } \\
g=2, & n=m_{1}+m_{2}, m_{1} \text { or } m_{2} \text { is odd. } \\
g=3, & \left(m_{1}, m_{2}\right)=(1,1), N^{3}=\frac{S O(3)}{\mathbb{Z}_{2}+\mathbb{Z}_{2}}, \\
g=4, & \left(m_{1}, m_{2}\right)=(1, k), N^{2 k+2}=\frac{S O(2) \times S O(k+2)}{\mathbb{Z}_{2} \times S O(k)},(k \geq 1), \\
g=6, & \left(m_{1}, m_{2}\right)=(1,1), N^{6}=\frac{S O(4)}{\mathbb{Z}_{2}+\mathbb{Z}_{2}} .
\end{array}
$$

\section{Relation to FOOO theory}

Fukaya-Oh-Ohta-Ono deeply investigate the Floer theory on Lagrangian intersections ([14]). We note some parts related to our work.

Theorem 8.1 ([14], Theorem H). Let $L$ be a closed Lagrangian submanifold in a closed symplectic manifold $M$, and let $L$ be spin (or, more generally, relatively spin). If the natural map $H_{\star}(L, \mathbb{Q}) \rightarrow H_{\star}(M, \mathbb{Q}$ ) is injective, then for any Hamiltonian diffeomorphism $\varphi$ such that $L$ is transversal to $\varphi(L)$, it holds

$$
\sharp(L \cap \varphi(L)) \geq \sum_{k} \operatorname{rank} H_{k}(L, \mathbb{Q}) .
$$

Because they use the $\mathbb{Q}$-coefficient, it is important to know if the moduli space $\mathcal{M}(x, y)$ is orientable or not. Since we used $\mathbb{Z}_{2}$-coefficient, we needed not to care about that point.

However, it is an interesting question whether the Gauss image $L$ of an isoparametric hypersurface is spin or not. This is completely determined by Z.Z. Tang. 
Proposition $8.2([36])$. The existence of a spin structure on its Gauss image $L^{n}=\mathcal{G}\left(N^{n}\right)$ is as follows:

\begin{tabular}{|c|c|c|}
\hline$g$ & $\left(m_{1}, m_{2}\right)$ & $L$ \\
\hline 1 & $n$ & spin \\
\hline 2 & $n:$ even & spin \\
& $n:$ odd & not spin \\
\hline 3 & $m=1,2,4,8$ & spin \\
\hline 4 & $(1,1)$ & spin \\
& $m_{1}+m_{2} \geq 3$ : odd & not relatively spin \\
& otherwise & not spin \\
\hline 6 & $(1,1)$ & spin \\
& $(2,2)$ & not spin \\
\hline
\end{tabular}

From this result, at least when $g=4$ and $m_{1}+m_{2} \geq 3$ is odd, we cannot apply FOOO's result with $\mathbb{Q}$ coefficient, even if the other conditions are satisfied.

Although $L$ is spin when $\left(m_{1}, m_{2}\right)=(1,1)$, we do not know yet the homology $H_{\star}(L, \mathbb{Q})$, nor $H_{\star}(L, \mathbb{Q}) \rightarrow$ $H *\left(Q_{n}(\mathbb{C}), \mathbb{Q}\right)$ is injective or not. Our next task is to consider these problems.

By the way, any isoparametric hypersurface $N^{n}$ in the standard sphere $S^{n+1}$ is a spin manifold.

\section{Open problems and conjecture}

\section{Problems.}

1. Determine $H F(L)$ for $g=3, m=1$.

2. When $g=4$ and $\left(m_{1}, m_{2}\right)=(1, k)$, is $L$ Hamiltonian non-displaceable?

3. When $g=6$ and $m=1$, is $L$ Hamiltonian non-displaceable?

4. Determine $H F(L)$ for all the remaining cases.

5. Consider the Lagrangian intersection for any two $L_{1}, L_{2}$.

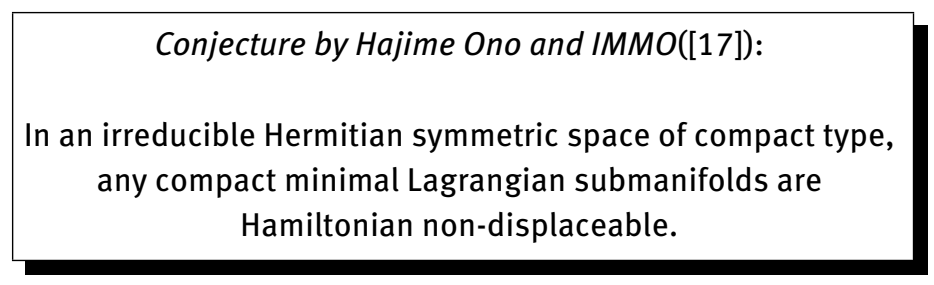

Acknowledgements. The article is based on a talk of the authors at the 5th workshop "Complex Geometry and Lie Groups"(Florence, Italy, June 11-15, 2018). The authors sincerely would like to thank the organizers, especially Professors Anna Fino, Fabio Podestà, Keizo Hasegawa and Ryushi Goto for their kind invitations and warm hospitality. The authors also would like to thank the referee for careful reading and valuable suggestions.

The authors were supported by JSPS KAKENHI Grant Numbers JP15H03616, JP18K03307, JP18H03668, JP17H06127.

\section{References}

[1] P. Albers, On the extrinsic topology of Lagrangian submanifolds, International Mathematical Research Notices 38 (2005), 2342-2371. 
[2] P. Biran, Lagrangian non-intersections, Geom. Funct. Anal. 16 (2006), 279-326.

[3] É. Cartan, Sur des familles remarquables d'hpersurfaces isoparamétriques dans les espaces sphériques, Math. Z. 45 (1939), 335-367.

[4] T. Cecil, Q.S. Chi and G. Jensen, Isoparametric hypersurfaces with four principal curvatures, Ann. Math., 166 (2007), 1-76.

[5] Q.S. Chi, Isoparametric hypersurfaces with four principal curvatures, II, Nagoya Math. J., 204, (2011), 1-18.

[6] Q.S. Chi, Isoparametric hypersurfaces with four principal curvatures, III, , J. Differential Geom., 94, (2013), 3: 469-504.

[7] Q.S. Chi, Isoparametric hypersurfaces with four principal curvatures, IV, to appear in J. Differential Geom., (2019).

[8] K. Cieldick and E. Goldstein, A note on the mean curvature, Maslov class and symplectic area of Lagrangian immersions J. Symplectic Geom. 2 (2004), no. 2, 261-266.

[9] M. Damian, Floer homology on the universal cover, Audin's conjecture and other constrains on Lagrangian submanifolds, Comment. Math. Helv. 87 (2012), 433-462.

[10] J. Dorfmeister and E. Neher, Isoparametric hypersurfaces, case $g=6, m=1$, Comm. Algebra 13 (1985), 2299-2368.

[11] D. Ferus, H. Karcher and H. F. Münzner, Cliffordalgebren und neue isoparametrische Hyperflächen, Math. Z. 177 (1981),479502.

[12] A. Floer, Morse theory for Lagrangian intersections, J. Differential Geom. 28 (1988), 513-547.

[13] A. Floer, A relative Morse index for the symplectic action, Commun. Pure Appl. Math. 41 (1988), 393-407.

[14] K. Fukaya, Y.G. Oh, H. Ohta and K. Ono, 'Lagrangian Intersection Floer Theory, Anormaly and Obstruction, Part I and II AMS (2009).

[15] W.-Y. Hsiang and H.B. Lawson, Minimal submanifolds of low cohomogeneity, J. Differential Geom. 5 (1971), no. 1-2, 1-38.

[16] H. Iriyeh, Symplectic topology of Lagrangian submanifolds of $\mathbb{C} P^{n}$ with intermediate minimal Maslov numbers, Adv. Geom. 17 (2017), no. 2, 247-264.

[17] H. Iriyeh, H. Ma, R. Miyaoka and Y. Ohnita. Hamiltonian non-displaceability of Gauss images of isoparametric hypersurfaces, Bull. London Math. Soc. 48 (5) (2016):802-812.

[18] H. Iriyeh, T. Sakai and H. Tasaki, Lagrangian Floer homology of a pair of real forms in Hermitian symmetric spaces of compact type, J. Math. Soc. Japan 65 (2013), 1135-1151.

[19] H. Ma and Y. Ohnita, On Lagrangian submanifolds in complex hyperquadrics and isoparametric hypersurfaces in spheres, Math. Z. 261 (2009), 749-785.

[20] H. Ma and Y. Ohnita, Differential geometry of Lagrangian submanifolds and Hamiltonian variational problems, Harmonic maps and differential geometry, Contemp. Math., 542, 115-134 Amer. Math. Soc., Providence, RI, 2011.

[21] H. Ma and Y. Ohnita, Hamiltonian stability of the Gauss images of homogeneous isoparametric hypersurfaces. I, J. Differential Geom. 97 (2014), 275-348.

[22] H. Ma and Y. Ohnita, Hamiltonian stability of the Gauss images of homogeneous isoparametric hypersurfaces. II, Tohoku Math. J. 67 (2015), 195-246.

[23] R. Miyaoka, Isoparametric hypersurfaces with $(g, m)=(6,2)$, Ann. of Math. (2) 177 (2013), 53-110.

[24] R. Miyaoka, Errata of isoparametric hypersurfaces with $(g, m)=(6,2)$ Ann. of Math. (2) 183 (2016) 1057-1071.

[25] H. F. Münzner, Isoparametrische Hyperflächen in Sphären, and II, Math. Ann. 251 (1980), 57-71, 256 (1981), $215-232$.

[26] Y.G. Oh, Floer cohomology of Lagrangian intersections and pseudo-holomorphic discs I, Comm. Pure Appl. Math. 46 (1993), 949-994; Addendum 48 (1995) 1299-1302.

[27] Y.G. Oh, Floer cohomology of Lagrangian intersections and pseudo-holomorphic discs III, H. Hofer, et al. (Eds.), The Floer Memorial Volume, Birkhäuser, Basel, 1995, 555-573.

[28] Y.G. Oh, Floer cohomology, spectral sequences, and the Maslov class of Lagrangian embeddings, Int. Math. Res. Not. 7 (1996), 305-346.

[29] Y. Ohnita, Geometry of Lagrangian submanifolds and isoparametric hypersurfaces Proceedings of the 14th International Workshop on Differential Geometry and the 3rd KNUGRG-OCAMI Differential Geometry Workshop 14, 43-67, Natl. Inst. Math. Sci. (NIMS), Taejŏn, 2010.

[30] Y. Ohnita, Geometry of Lagrangian submanifolds in complex hyperquadrics and the Gauss images of isoparametric hypersurfaces Proceedings of the 19th International Workshop on Hermitian-Grassmannian Submanifolds and 10th RIRCM-OCAMI Joint Differential Geometry Workshop, 283-309, Natl. Inst. Math. Sci. (NIMS), Taejŏn, 2015.

[31] Y. Ohnita, On Floer homology of the Gauss images of isoparametric hypersurfaces. Hermitian-Grassmannian submanifolds, 235-247, Springer Proc. Math. Stat., 203, Springer, Singapore, 2017.

[32] H. Ono, Integral formula of Maslov index and its applications Japan J. Math., 30 no. 2, (2004), 413-421.

[33] H. Ozeki and M. Takeuchi, On some types of isoparametric hypersurfaces in spheres I and II, Tohoku Math. J. 27 (1975), 515-559, 28 (1976), 7-55.

[34] B. Palmer, Hamiltonian minimality and Hamiltonian stability of Gauss maps, Diff. Geom. and its Appl. 7 (1997), 51-58.

[35] R. Takagi and T. Takahashi On the principal curvatures of homogeneous hypersurfaces in a sphere, Differential Geom. in honor of Kentaro Yano (1972), 469-481.

[36] Z.Z. Tang, private communication (2018).

[37] C. Viterbo, Intersection de sous-varietes Lagrangiennes, fonctionelles d'action et indice des systemes Hamiltoniens, Bull. Soc. Math. Fr. 115, 361-390 (1987) 